\title{
EFECTO DEL EMPAQUE SOBRE LA TEXTURA Y EL COLOR DEL CAMOTE (Ipomoea batatas L.) DURANTE EL PROCESO DE “CURADO"1
}

\author{
Alejandro Chacón-Villalobos ${ }^{2}$, Yury Reyes-Cruz ${ }^{3}$
}

\begin{abstract}
RESUMEN
Efecto del empaque sobre la textura y el color del camote (Ipomoea batatas $\mathrm{L}$.) durante el proceso de "curado". El presente estudio se efectuó en la Escuela de Tecnología de Alimentos de la Universidad de Costa Rica entre junio y diciembre del 2006. Camotes (Ipomea batatas L.) adquiridos cuatro horas post cosecha en el Centro Nacional de Abastecimiento y Distribución de Alimentos (CENADA), fueron lavados y preseleccionados, luego aleatoriamente distribuidos en cinco grupos experimentales, identificándolos individualmente y determinando su peso. Al grupo control se le determinó la calidad general con una escala visual, la fuerza de penetración $\left(\mathrm{kg} / \mathrm{cm}^{2}\right)$ y el color (cáscara y pulpa), con base en la escala CIELAB. El primer grupo se empacó con una película plástica microperforada, el segundo en polietileno de baja densidad y el tercero no se empacó. Los tres se incubaron siete días a $29{ }^{\circ} \mathrm{C}$. El cuarto grupo permaneció sin empaque a la intemperie el mismo período. Pasado ese tiempo todos los grupos experimentales se evaluaron para peso, apariencia, color y textura. Se realizó un análisis de varianza entre tratamientos seguido de una evaluación de medias de Tukey. La fuerza promedio de penetración fue mayor ( $\mathrm{p}<0,05)$ para los camotes empacados con polietileno de baja densidad y para los expuestos al ambiente. No se evidenciaron diferencias significativas en el color de la pulpa entre tratamientos. Con respecto al resto de tratamientos, para la cáscara hubo diferencias significativas, las más brillantes fueron almacenados en empaques, comparados con los almacenados sin protección.
\end{abstract}

Palabras clave: Vida útil, película, atmósfera modificada, color, textura.

\begin{abstract}
Effect of packing upon texture and color of sweet potatoes (Ipomoea batatas L.) during the "curing" process. The evaluation was conducted at the School of Food Technology of the University of Costa Rica (San José) between June and December 2006. Sweet potatoes (Ipomea batatas L.) acquired four hours after harvest from the Centro Nacional de Abastecimiento y Distribución de Alimentos (CENADA), were washed, pre-selected, and randomly grouped in five experimental blocks after individual identification and weighing. The control group was evaluated for general quality using a visual scale, penetration force $\left(\mathrm{kg} / \mathrm{cm}^{2}\right)$ and color (peel and pulp) using the CIELAB scale. The first group was packed with a micro perforated film, and the second group with low-density polyethylene (LDP); group three was not packed. The three groups were stored for seven days at $29^{\circ} \mathrm{C}$. The fourth group remained unpacked at room temperature for the same period. After the storage treatment, all groups were evaluated for visual appearance, texture, color and weight ANOVA tests followed by Tukey comparisons were conducted. Medium penetration force was higher $(\mathrm{p}<0.05)$ for samples packed with LDP and for samples held at room temperature. Pulp color did not differ among treatments. The shiniest peels were obtained from sweet potatoes packed in low-permeability foils.
\end{abstract}

Key words: Shelf life, plastic wrap, modified atmosphere, color, texture.

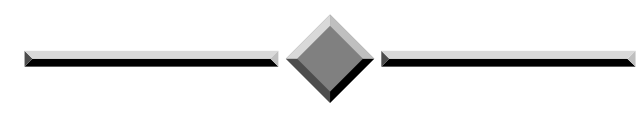

\footnotetext{
Recibido: 14 de noviembre, 2007. Aceptado: 20 de marzo, 2009.

2 Estación Experimental Alfredo Volio Mata, Facultad de Ciencias Agroalimentarias, Universidad de Costa Rica. San José, Costa Rica. alejandro.chacon@ucr.ac.cr

3 Escuela de Tecnología de Alimentos, Facultad de Ciencias Agroalimentarias, Universidad de Costa Rica. San José, Costa Rica. quimico02@ hotmail.com
} 


\section{INTRODUCCIÓN}

El camote (Ipomea batatas L.) es un importante cultivo tropical y subtropical perteneciente a la familia de las Convolvuláceas, el cual exhibe diversos ecotipos (Kay 1973, Collins y Abdul Aziz 1982, Sweet Potato First International Symposium 1982, Saiful Islam et al. 2002). Es reconocido por su robustez, y por su resistencia a la sequía, a las pestes y a las enfermedades (Noda et al. 1992). Se caracteriza por contar con elongaciones carnosas y almidonosas que constituyen tubérculos de naturaleza biológicamente única (Kay 1973, Hoover et al. 1983, Tanaka et al. 2005). Estos constituyen una importante fuente de vitaminas A y C, minerales, energía, fibra dietética y alguna proteína (Collins y Washam-Hutsell 1987, Collins et al. 1995, Lerner 2005); además de azúcares simples (Huang et al. 1999).

El callo externo del peridermo contiene cantidades variables de antocianinas de moderada estabilidad, las cuales poseen actividad antioxidante y antimutagénica, siendo así útiles en la industria agroalimentaria no solo por su capacidad colorante sino por su valor nutracéutico (Terahara et al. 2000). El área comprendida entre el peridermo y el cambium (lacticifer), la ocupan aproximadamente $5 \mathrm{~mm}$ de tejido compuesto por parénquima pobre en almidón, además de una serie de células ricas en látex y que se hacen más numerosas en las cercanías del cambium. Este último cuenta a la vez con una serie de elementos del xilema radialmente ordenados junto con células del parénquima repletas de almidón (Walter y Schadel 1982). Una activa división celular y expansión en los meristemos localizados en el xilema, dan como resultado un aumento en el grosor del tubérculo (Tanaka et al. 2005). El color interno de este tejido puede variar desde blanco amarillento hasta anaranjado o incluso morado entre diferentes cultivares (Bicudo de Almeida-Muradian et al. 1992, Instituto Nacional de Investigación y Extensión Agrícola 2008).

Los camotes son tubérculos perecederos, y no suelen ser almacenados por periodos muy prolongados sin antes sufrir algún tipo de acondicionamiento (Kay 1973). La vida de mercado de un camote fresco no acondicionado es usualmente menor a aquellos que han sido curados, variando esto según el manejo posterior y la misma variedad del tubérculo (Quirien et al. 2003). La conservación a bajas temperaturas es inviable, dados los daños mecánicos por frío que se produce cuando se almacena por debajo de $\operatorname{los} 10{ }^{\circ} \mathrm{C}$ (Kay 1973, Afeck et al. 1998). La formación de manchas pardas, que es uno de los principales ejemplos de deterioro, es común en camotes cortados crudos o con heridas en el peridermo, lo cual es causado esencialmente por la acción de la enzima polifenoloxidasa al contacto con el oxígeno ambiental (Walter y Schadel 1982). Las dificultades asociadas con el manejo de camotes no acondicionados representan una problemática importante, dado que mucha de la industria de los derivados del camote requiere de una disponibilidad anual del producto, sin importar la estacionalidad particular de la región (Picha 1986, Quirien et al. 2003).

Algunos procesos de acondicionamiento y conservación implican el lavado, desinfección y posterior tratamiento con agentes como el $\mathrm{Na}_{2} \mathrm{CO}_{3}, \mathrm{Na}_{3} \mathrm{PO}_{4} \mathrm{O}$ con fungicidas que empleen iprodione como agente activo, lo que evita la proliferación de hongos causantes de deterioro y toxicidad como Rhizopus spp. o Fusarium spp. (Kay 1973, Walter et al. 1993, Afeck et al. 1998).

Con la intención de facilitar el almacenamiento, de prevenir y sanar heridas en el peridermo y el cambium, y de desarrollar un mejor sabor, los camotes se someten casi de manera inmediata después de su cosecha a un tratamiento denominado curado (Hoover et al. 1983, Lerner 2005), que consiste en almacenar los camotes a una temperatura de alrededor de $30{ }^{\circ} \mathrm{C}$, y a una humedad relativa de $80 \%-95 \%$ por un periodo de cinco a 10 días (Hoover et al. 1983, Lerner 2005, LSU AG Center 2005, James 2005, Grandberry et al. 2005). El proceso estimula la formación de una capa protectora de corcho, suberina y otros materiales cerosos sobre la superficie del tubérculo, generándose una barrera física protectora que aminora la pérdida de humedad y el ataque fúngico y bacteriano (Motes y Criswell 2005). Después del curado, los camotes pueden ser almacenados exitosamente para su preservación transitoria a temperaturas entre $13{ }^{\circ} \mathrm{C}-16^{\circ} \mathrm{C}$, con una humedad relativa que varía entre $85 \%$ - 90 $\%$ (Kay 1973, Hoover et al. 1983, Lerner 2005, LSU AG Center 2005, James 2005, Grandberry et al. 2005, Motes y Criswell 2005).

Durante el curado se origina además una rápida formación de la enzima $\alpha$-amilasa, la cual en unión con la acción de la $\beta$-amilasa, provoca la conversión del almidón y de los carbohidratos estructurales en azúcares simples, aumentando consecuentemente el sabor dulce y ablandándose los tejidos (Hoover et 
al. 1983, McArdle y Bouwkamp 1986, Szyperski et al. 1986, Lerner 2005). A estas enzimas se suma la acción de invertasas y sucrosa sintetasas, que también influyen en los niveles de azúcares en los tubérculos durante el almacenamiento (Huang et al. 1999). La actividad enzimática endógena del camote varía grandemente según sea la época en que fue cosechado y la técnica de curado, por lo cual el control de la hidrólisis del almidón es de difícil implementación (Szyperski et al. 1986). A lo largo de todo el proceso, el porcentaje de humedad cambia de manera poco significativa, a medida que el contenido de pectina soluble aumenta al igual que lo hace el nitrógeno no proteico (Heinze y Appleman 1943). Como resultado de los procesos descritos, la incidencia de problemas de alta dureza en el camote tiende a reducirse sustancialmente (Broadus et al. 1980).

La utilización de películas de empaque con tasas apropiadas de transmisión de gases, y que generen atmósferas modificadas en combinación con almacenamientos a bajas temperaturas, suelen ser una buena forma de conservación de los vegetales frescos (Singh y Heldman 1998). McConnell et al. (2005) reportan que para camotes rebanados, el uso de empaques de moderada permeabilidad al oxígeno $\left(7.000 \mathrm{~cm}^{3} / \mathrm{atm} /\right.$ $\mathrm{m}^{2} / 24 \mathrm{~h}$ ) generan menores cambios en la firmeza y otros atributos sensoriales y fisicoquímicos en comparación con el almacenamiento al aire libre.

Son escasas las fuentes documentales que señalan el efecto del empaque en atmósferas modificadas, durante el proceso de curado de los camotes. Algunos autores como Chang et al. (1983), señalan que en condiciones de bajo contenido de $\mathrm{O}_{2}$, el contenido interno de gases en el camote es rápidamente reemplazado por $\mathrm{CO}_{2}$, aumentándose la actividad de enzimas como la piruvato descarboxilasa y la producción de etanol. Es posible esperar que en condiciones donde existe cierta disponibilidad de oxígeno y un contenido apropiado de etileno, el camote presente un importante incremento en su tasa de respiración (Reid y Pratt 1972, Dizengremel y Lance 1976).

El objetivo de este trabajo fue estudiar el efecto del empaque en diferentes películas sobre el color interno y externo, el peso, la textura del área entre el peridermo y el cambium, y la apariencia general, de camotes recién cosechados y almacenados en diferentes condiciones de temperatura que emulan a las del proceso de curado.

\section{MATERIALES Y MÉTODOS}

\section{Preparación y evaluación de las muestras}

El estudio se efectuó entre los meses de junio y diciembre de 2006, en las instalaciones del Laboratorio de Bromatología de la Escuela de Tecnología de Alimentos de la Universidad de Costa Rica (ETA), ubicadas en San Pedro de Montes de Oca, San José, Costa Rica.

El procedimiento experimental general a describir seguidamente se repitió dos veces. Para cada una de esas dos corridas experimentales efectuadas con un mes de diferencia, se adquirió un lote de 40 $\mathrm{kg}$ de camote cuatro horas después de la cosecha. La adquisición se efectuó en el Centro Nacional de Abastecimiento y Distribución de Alimentos (CENADA), localizado en la provincia de Heredia, a una distancia de $12,5 \mathrm{~km}$ de la ETA. Se supervisó que el lote no fuera expuesto a la intemperie ni al daño mecánico por manipulación, durante las cuatro horas transcurridas desde la cosecha hasta su envío a la ETA.

Los camotes fueron lavados individualmente con agua potable para eliminar impurezas y suciedad. Se seleccionaron en ese momento, aquellos tubérculos que no presentaran daños aparentes. Las unidades preseleccionadas fueron distribuidas de manera aleatoria en cinco grupos experimentales integrados cada uno por 10 camotes de peso y forma similar. Seguidamente se identificaron individualmente por medio de una numeración, y se pesaron en una balanza granataria electrónica Sartorius modelo cp6201. Las piezas sobrantes, una vez formados los grupos, fueron descartadas y donadas para su empleo en nutrición animal.

El primer grupo se empacó individualmente en bolsas de $25 \mathrm{~cm}$ x $30 \mathrm{~cm}$ empleando una película de poliolefina orientada microperforada Cryovac ${ }^{\circledR}$ SM-25 que se selló con calor. El segundo grupo se empacó de la misma forma en bolsas de iguales dimensiones, pero constituidas por una película de polietileno de baja densidad (LDPE), a las que se les hizo dos orificios de un diámetro aproximado de $1,7 \mathrm{~mm}$ en el punto central de cada cara de la bolsa, con la intención de permitir un intercambio gaseoso. Los grupos 3, 4 y 5 no recibieron empaque alguno.

El primero, segundo y tercer grupo se colocaron en incubadoras Precision Scientific Incubator 815 para las cuales se ajustó la temperatura a $29{ }^{\circ} \mathrm{C}$. Las muestras fueron mantenidas en la incubadora cerrada durante un 
período de siete días. Se emuló así las condiciones de curado, generando las temperaturas por medio de la incubadora y las humedades relativas características (85 $\%-100 \%$ ), por medio del empaque en los grupos que lo poseían. El tercer grupo experimental sirvió entonces para evaluar el efecto de la incubación carente de empaque. La temperatura $\left(29^{\circ} \mathrm{C}\right)$ y la humedad relativa se controlaron a lo largo del periodo de almacenamiento, tanto en el interior de la cámara como en el interior de los empaques, por medio de termohigrómetros calibrados Radio Shack Thermo Hygro ${ }^{\circledR}$ modelo 63-1032.

El cuarto grupo experimental se mantuvo almacenado sin empaque y a temperatura ambiente $\left(25^{\circ} \mathrm{C}\right)$, en una habitación destinada para tal efecto durante el mismo periodo.

El quinto grupo experimental, que constituyó el grupo control, no sufrió almacenamiento ni empaque alguno, y fue evaluado inmediatamente después del lavado y preselección de manera visual e instrumental para apariencia, color y fuerza de penetración, empleando las metodologías que se describirán más adelante en este procedimiento.

Finalizado el lapso de almacenamiento, cada una de las piezas pertenecientes a los diferentes tratamientos se pesó nuevamente con la finalidad de evidenciar variaciones de peso durante el periodo. Se evaluó seguidamente y en forma visual la calidad general de todas las piezas según tratamiento enfocándose en aspectos como el color general, la textura al tacto, y la turgencia de la cáscara, todo ésto con la intención de determinar posibles defectos físicos o microbiológicos evidentes debidos al proceso. Esta evaluación aparente fue efectuada por tres investigadores con experiencia basándose en un cuadro de atributos de cinco puntos para cada uno de los tres aspectos antes citados donde un valor de uno correspondió a un color muy opaco, una textura muy blanda y una turgencia muy pobre.

Se valoró instrumentalmente a continuación la fuerza requerida para lograr la penetración de la corteza de cada una de las 10 piezas integrantes de los cuatro grupos experimentales, empleando un texturómetro Stable Micro Systems ${ }^{\circledast}$ modelo TA-XT plus para una celda de $50 \mathrm{~kg}$ en modo "Peak" y una penetración total de $5 \mathrm{~mm}$.

En la evaluación de la penetración de la corteza, para cada pieza se efectuaron 10 repeticiones de la medición de la fuerza de penetración en posiciones aleatoriamente selecionadas a lo largo del cuerpo del tubérculo, lo cual suma un total de 100 mediciones de la fuerza de penetración para cada grupo experimental. Posteriormente, los camotes fueron cortados transversalmente en dos mitades, evaluándose el color interno y el color de la cáscara por medio de un colorímetro HunterLab modelo ColorFlex ${ }^{\circledR}$ de la escala de CIELAB. Esta escala es un modelo cromático tridimensional que describe todos los colores que puede percibir el ojo humano, y que está compuesto por tres variables: un valor L que señalan la luminosidad del color (rango entre $\mathrm{L}=0$ para negro y $\mathrm{L}=100$ para blanco), un valor a que define si el color está entre magenta y verde (valores negativos tienden a verde, positivos a magenta), y un valor $\mathbf{b}$ que define la posición entre amarillo (-) y azul (+) (Hill et al. 1997). Para cada pieza se efectuaron cuatro evaluaciones de la medición de color empleando esta escala en posiciones aleatoriamente seleccionadas tanto para color de la cáscara como para color interno, sumando así un total de 40 valoraciones por grupo experimental. Se empleó la relación de las variables a/b para establecer que tan rojo o amarillo es el camote inicialmente tanto en cáscara como en cuanto al color interno y su cambio al final del lapso de tratamiento. Si el valor a/b es alto, ya sea en la cáscara o a lo interno, indica que el color del tejido tiende a ser más rojo y si es bajo indica que el color es más amarillo. Esta relación a/b fue utilizada por Pangloli et al. (2000) y Collins et al. (1995) en camotes procesados.

\section{Análisis estadístico}

Completadas las corridas experimentales, se realizó un análisis de varianza empleando un diseño irrestricto al azar para determinar diferencias significativas entre los tratamientos para las variables evaluadas de porcentaje de pérdida de peso, color y fuerza de penetración, utilizando una evaluación de medias de Tukey. El error de muestreo alfa utilizado en el experimento fue de 0,05.

\section{RESULTADOS Y DISCUSIÓN}

\section{Evaluación de la calidad aparente una vez finaliza- do el almacenamiento}

Al evaluar la apariencia general de los camotes de los diferentes grupos experimentales, se notó como los camotes almacenados en la cámara sin empaque presentaron una ligera disminución en intensidad de color comparados con aquellos que estuvieron empacados. 
La baja humedad del interior de la cámara $(52,1 \%)$ provocó en apariencia un evidente efecto de desecado de la cáscara. Si bien es cierto, este efecto se determinó también en menor medida para los camotes almacenados sin empaque a la intemperie, en este caso la humedad relativa fue intermedia por lo cual la desecación no fue tan acentuada (Cuadro 1). Otras diferencias de color de la cutícula aparte de las mencionadas no fueron evidentes a simple vista por lo cual se hizo necesaria la evaluación instrumental.

Cuadro 1. Temperaturas y humedades relativas medias de almacenamiento de los camotes durante siete días. San José, Costa Rica. 2007.

\begin{tabular}{lcc}
\hline Tratamiento & $\begin{array}{c}\text { Temperatura } \\
\left({ }^{\circ} \mathbf{C}\right)\end{array}$ & $\begin{array}{c}\text { Humedad } \\
\text { relativa (\%) }\end{array}$ \\
\hline Sin almacenar sin empaque & 21,0 & 85,3 \\
Incubado sin empaque & 29,0 & 52,1 \\
$\begin{array}{l}\text { Incubado + micro-perforado } \\
\text { Incubado + polietileno de }\end{array}$ & 29,2 & 87,4 \\
baja densidad & 29,1 & 100,0 \\
\hline
\end{tabular}

En cuanto a la dureza al tacto se puede concluir que el tratamiento de polietileno de baja densidad y el de temperatura ambiente $\left(25^{\circ} \mathrm{C}\right)$, resultaron ser los tratamientos en que los camotes presentaron mayor dureza aparente en general, mientras los camotes almacenados en la cámara presentaron la menor. Esto concordará más adelante en este trabajo con las mediciones realizadas instrumentalmente.

En ninguno de los tratamientos se presentaron daños microbianos o fúngicos durante el periodo de curado de los camotes, razón por la cual se consideraron todas las piezas obtenidas como aptas para las evaluaciones instrumentales. Esto a la vez refleja que aunque se registraron altas humedades relativas en varios de los tratamientos (superior al 85\%), éstas no promovieron la aparición de mohos u otras causas de deterioro como las mencionadas por Motes y Criswell (2005), por lo cual no se considera que los tratamientos puedan presentar esta problemática al ser efectuados en piezas debidamente lavadas previamente como en este caso.

\section{Temperatura y humedad relativa}

En el Cuadro 1 se presentan las temperaturas y humedades relativas de almacenamiento promedio, calculadas a partir de los valores medidos con los termohigrómetros a lo largo del periodo de almacenamiento para los diferentes tratamientos.

En las incubadoras fue posible mantener la temperatura deseada de $29^{\circ} \mathrm{C}$, a lo largo del período de estudio, temperatura que es similar a aquella dónde se registra el curado (Cuadro 1). Los valores de humedad relativa obtenidos también son consistentes con lo esperado, al ser éstos más altos en tanto más bajo es el intercambio gaseoso que permite la película plástica de empaque, existiendo más intercambio en el microperforado que en el polietileno. Lo anterior es también sinónimo de la formación de una atmósfera modificada, particular en el interior de cada uno de los empaques, dónde podría esperarse que el proceso de respiración del tubérculo, y por ende muchos cambios fisicoquímicos asociados, se vean disminuidos entre mayor sea la humedad relativa y menor el intercambio gaseoso con el exterior dada la menor disponibilidad de oxígeno en el interior (Schippers 1977). En un curado convencional donde no hay empacado ésto no debería registrarse.

El microperforado emuló, dentro de la cámara, una humedad relativa que se asemeja a la experimentada en el medio ambiente, con la diferencia de que es esperable una menor fluctuación de esta variable dentro del empaque microperforado y que, dentro de una cámara se puede estandarizar asimismo la temperatura. La humedad relativa más baja fue la registrada dentro de la incubadora, aspecto que concuerda también con lo esperado.

Según algunos estudios y como se indicó antes, el curado de los camotes consiste en someterlos a condiciones de $29-30{ }^{\circ} \mathrm{C}$ y $80-95 \%$ HR durante cinco a siete días (Wang et al. 1998, Buescher et al. 1975, Mukerjee y Prasad 1972). Según lo expuesto en el Cuadro 1, con los empaques fue posible simular en esta experiencia las condiciones de humedad relativa requeridas para el proceso de curado. Por esta razón podría ser factible el emplear empaques como una opción dónde no se cuente con la capacidad de regular la humedad relativa pero si la temperatura, dejando que sean las películas las que lo hagan de manera pasiva. Dado que James (2005), especifica que las condiciones de curado se pueden incluso dar a temperaturas superiores a los 21 ${ }^{\circ} \mathrm{C}$, con base a las condiciones experimentales estudiadas, aún el tratamiento a temperatura ambiente (25 $\left.{ }^{\circ} \mathrm{C}\right)(85,31 \% \mathrm{HR})$ pudo haber generado algún grado de curado. 


\section{Pérdidas de peso}

Según Quirien et al. (2003), la vida útil del camote está estrechamente relacionada con la pérdida de peso, siendo de hecho la principal causa de deterioro. Estimaciones teóricas reportadas por Wills et al. (1998), Singh y Heldman (1998) y De la Asunción (2005) ${ }^{4}$, para camotes almacenados sin empaque la pérdida de peso promedio en un periodo de siete días se estima en $4,5 \%$ para una humedad relativa de $87,44 \%$ y una temperatura de $29,21^{\circ} \mathrm{C}$. Por otro lado para una humedad relativa de $100 \%$ y una temperatura de $29,1{ }^{\circ} \mathrm{C}$, la pérdida promedio considerada es de 5,6 \% .

En el Cuadro 2 se muestra la pérdida porcentual promedio de peso en los diferentes tratamientos experimentales calculada a partir de los pesos iniciales y finales de los camotes.

Cuadro 2. Pérdida de peso promedio, experimentada en los diferentes tratamientos de acondicionamiento y conservación de camote, evaluados después de siete días de almacenamiento. San José, Costa Rica. 2007.

\begin{tabular}{lc}
\hline Tratamiento & Pérdida de peso $(\%)^{*}$ \\
\hline Almacenado sin empaque & $7,9 \mathrm{a}$ \\
Incubado sin empaque & $20,4 \mathrm{~b}$ \\
Incubado + micro-perforado & $5,3 \mathrm{a}$ \\
Incubado + polietileno de & $1,5 \mathrm{c}$ \\
baja densidad & \\
\hline
\end{tabular}

"Los datos seguidos de una misma letra no son estadísticamente diferentes $(p>0,05)$, medias Tuckey.

Los resultados obtenidos son consistentes con lo esperado teóricamente. Es posible ver como la pérdida de peso se incrementó a medida que el camote tuvo una mayor posibilidad de intercambiar gases y humedad con su medio circundante. Esto es especialmente evidente en los casos extremos, donde el polietileno permitió una pérdida de peso de sólo el 1,5\%. En el caso del camote expuesto sin protección al medio de baja humedad re-

4 De la Asunción, R. 2005. Cálculos de almacenamiento de vegetales frescos. Escuela de Tecnología de Alimentos de la Universidad de Costa Rica. San José. Consulta personal. lativa de la cámara (alrededor de 52,1\% de humedad relativa) se manifiesta un $20,4 \%$ de pérdida.

La menor pérdida de peso que aconteció en el tratamiento de camotes empacados en polietileno de baja densidad $(1,5 \%)$, fue significativamente diferente $(\mathrm{p}<0,05)$ a las pérdidas obtenidas en el resto de los tratamientos. En este caso la alta humedad relativa de la atmósfera modificada que se generó, combinada con la disminución mayor de la tasa de respiración del camote, explicaría el resultado obtenido.

Entre los tratamientos "microperforado" y "ambiente" no se encontró diferencia significativa ( $\mathrm{p}>$ 0,05 ) en el porcentaje de pérdida de peso, lo cual está probablemente asociado con la similitud en cuanto a los valores de humedad relativa que ambos ambientes presentaron entre sí. Aunque el análisis estadístico no arrojó una diferencia significativa, si es posible visualizar que para el camote expuesto al medio ambiente existió una tendencia a que el valor de la pérdida de peso sea mayor, lo cual podría especularse, según los fundamentos teóricos, estaría asociado a una respiración relativamente más acentuada del tubérculo al encontrarse una mayor disponibilidad de oxígeno y no ser sujeto del efecto de la atmósfera modificada.

El tratamiento que presentó la mayor pérdida de humedad $(p<0,05)$ fue aquel dónde los camotes se conservaron en almacenamiento sin empaque en la cámara de incubación. Esto encuentra su explicación en la alta temperatura y la baja humedad relativa experimentada, lo cual provocó una migración de la humedad desde la matriz del camote al medio circundante.

Para las condiciones de temperatura y humedad relativa en el tratamiento del polietileno de baja densidad (humedad relativa de $100 \%$ y una temperatura de $29,1^{\circ} \mathrm{C}$ ) las fuentes antes citadas estimaron que la pérdida sin la utilización del empaque sería de 5,6 \%, mientras que el uso de éste durante el experimento al generar sólo una pérdida del 1,5\% representó un $4 \%$ de retención del peso.

\section{Fuerza de penetración}

En el Cuadro 3 se presentan los resultados de la fuerza de penetración promedio expresada en Newtons, obtenida para los diferentes grupos de camotes al final del periodo de siete días de almacenamiento.

Existió una mayor fuerza promedio de corte, en el tratamiento empacado en polietileno de baja densidad, mostrando diferencias significativas $(\mathrm{p}<0,05)$ con 
Cuadro 3. Fuerza promedio de penetración medida en los camotes en los diferentes tratamientos de acondicionamiento y conservación de camote, después de siete días de almacenamiento San José, Costa Rica. 2007.

\begin{tabular}{lc}
\hline Tratamientos & Fuerza de penetración $(\mathbf{N})^{*}$ \\
\hline Sin almacenar, sin empaque & \\
(control) & $9,29 \mathrm{a}, \mathrm{b}$ \\
Almacenado sin empaque & $9,50 \mathrm{a}, \mathrm{b}$ \\
Incubado sin empaque & $8,84 \mathrm{a}$ \\
Incubado + micro-perforado & $9,08 \mathrm{a}$ \\
Incubado + polietileno de & \\
baja densidad & $10,09 \mathrm{~b}$ \\
\hline
\end{tabular}

*Los datos seguidos de una misma letra no son estadísticamente diferentes $(\mathrm{p}>0,05)$.

respecto a los tratamientos colocados en la cámara y en el empaque microperforado, sin embargo, no existió diferencia significativa $(\mathrm{p}>0,05)$ con respecto al tratamiento colocado a temperatura ambiente $\left(25^{\circ} \mathrm{C}\right) \mathrm{ni}$ con respecto a los camotes pertenecientes al grupo de control. Al ser la respiración más limitada en la película de polietileno, dada la atmósfera modificada, podría suponerse que hay una menor actividad enzimática ligada a la respiración que disminuya la dureza, como es el caso de las amilasas (Hoover et al. 1983, McArdle y Bouwkamp 1986, Szyperski et al. 1986, Lerner 2005). Lo señalado por McConnell et al. (2005), con respecto a los pocos cambios en la dureza en empaques de atmósfera modificada podría estarse evidenciando, aunque los autores consideran que la menor pérdida de humedad tiene un efecto más importante. Lo anterior podría tener asidero en la tendencia que presenta el incubado sin empaque en presentar una menor dureza, dada su mayor pérdida de humedad y peso, aunque más investigación se necesita para corroborar esta afirmación.

No es posible afirmar, bajo las circunstancias experimentales evaluadas, que las condiciones de almacenamiento ensayadas alteraran significativamente la dureza de la corteza de los camotes del grupo de control, indiferentemente del tratamiento aplicado. No es posible afirmar por otro lado, que la simulación de un proceso de curado dentro de una atmósfera modificada tenga un efecto detrimental sobre la calidad textural del camote, por lo cual generar altas humedades relativas por medio del empaque en películas plásticas no está contraindicado para conservar las características texturales del tubérculo fresco. Este aspecto cobra importancia más adelante en este trabajo, al analizar el color y la calidad general de los tubérculos.

Aunque estos cambios en la dureza se hubiesen manifestado, escapando a la capacidad instrumental del equipo utilizado y siendo enmascarados por la variabilidad, para las variaciones observadas en este experimento (diferencias menores a $1 \mathrm{~N}$ ), es difícil afirmar que las mismas pudiesen generar un efecto sensorial considerable en las personas que consuman los camotes, por lo que su efecto, si lo hay, sería solo de valor técnico en el trasiego y manipulación preconsumo de los camotes.

Según Kay (1973), Lerner (2005) y Motes y Criswell (2005) durante el periodo de curado se forma una capa protectora especialmente en las heridas ocasionadas por la cosecha y que reduce la incidencia de infecciones fúngicas. Con base a la evidencia reportada, si ésta se generó en las pruebas efectuadas, su efecto textural no fue muy variable entre los tratamientos.

\section{Variaciones de color en pulpa y cáscara}

Los valores promedio para las variables $\mathrm{L}$ y $\mathrm{a} / \mathrm{b}$ (calculado este último partiendo de los valores a y b), y que fueron evaluados para las pulpas de los camotes pertenecientes a los diferentes grupos experimentales de acuerdo a la escala CIELAB, se presentan en el Cuadro 4.

Los resultados estadísticos obtenidos señalan que para las variables $\mathrm{L}$ y a/b no se pudieron establecer diferencias significativas $(p>0,05)$ entre ninguno de los tratamientos a los siete días después del almacenamiento. El color original manifestado en el grupo control, fue prácticamente el mismo que se registró en los tratamientos experimentales. Es posible afirmar entonces que los diferentes procesos de almacenamiento y empaque que se estudiaron no afectan el color interno de la pulpa del camote en un periodo de siete días posterior a la postcosecha, siendo el resultado el mismo si el camote se deja a la intemperie. La influencia del tipo de empaque fue mínima sobre el color interno de los camotes, el cual se mantuvo relativamente inalterable para los casos experimentales evaluados. No hubo un efecto negativo atribuible al empacado sobre esta variable. Se infiere que el color de la pulpa del camote presentó poca dependencia de las condiciones 
Cuadro 4. Variables de color promedio de la pulpa de los camotes, en los diferentes tratamientos de acondicionamiento y conservación de camote, después de siete días de almacenamiento. San José, Costa Rica. 2007.

\begin{tabular}{|c|c|c|c|c|}
\hline \multirow[t]{2}{*}{ Tratamientos } & \multicolumn{4}{|c|}{ Variables* } \\
\hline & $\mathbf{L}^{1}$ & & $\mathbf{y} \mathbf{b}^{3}$ & $\mathbf{a} / \mathbf{b}$ \\
\hline \multirow{2}{*}{$\begin{array}{l}\text { Sin almacenar, sin empaque } \\
\text { (control) }\end{array}$} & \multirow{2}{*}{86,78 a } & $\mathrm{a}$ & 2,01 & \multirow{2}{*}{$0,06 \mathrm{a}$} \\
\hline & & b & 31,63 & \\
\hline \multirow{2}{*}{ Almacenado sin empaque } & \multirow{2}{*}{87,49 a } & $\mathrm{a}$ & 3,30 & \multirow{2}{*}{$0,10 \mathrm{a}$} \\
\hline & & $\mathrm{b}$ & 32,13 & \\
\hline \multirow{2}{*}{ Incubado sin empaque } & \multirow{2}{*}{86,61 a } & $\mathrm{a}$ & 4,40 & \multirow{2}{*}{$0,14 \mathrm{a}$} \\
\hline & & $\mathrm{b}$ & 30,97 & \\
\hline \multirow{2}{*}{ Incubado + micro-perforado } & \multirow{2}{*}{87,55 a } & $\mathrm{a}$ & 3,90 & \multirow{2}{*}{$0,12 \mathrm{a}$} \\
\hline & & $\mathrm{b}$ & 31,30 & \\
\hline \multirow{2}{*}{$\begin{array}{l}\text { Incubado }+ \text { polietileno de } \\
\text { baja densidad }\end{array}$} & \multirow{2}{*}{$88,04 \mathrm{a}$} & $\mathrm{a}$ & 3,68 & \multirow{2}{*}{$0,11 \mathrm{a}$} \\
\hline & & $\mathrm{b}$ & 31,72 & \\
\hline
\end{tabular}

* Los datos seguidos de una misma letra no son estadísticamente diferentes $(\mathrm{p}>0,05)$.

${ }^{1}$ luminosidad del color, $0=$ negro y $100=$ blanco.

${ }^{2}$ color entre magenta y verde.

${ }^{3}$ color entre amarillo y azul.

de almacenamiento y empaque, en los primeros días postcosecha.

Los rangos de valores obtenidos concuerdan con los rangos de valores característicos obtenidos por $\mathrm{Bi}$ cudo de Almeida-Muradian et al. (1992) en su estudio de evaluaciones de color en la pulpa de seis variedades de camotes. Al parecer la pulpa de la variedad de camote estudiada fue muy estable al almacenamiento y al tiempo según los hallazgos expuestos.

Los valores promedio para las variables $\mathrm{L}$ y a/b para las cáscaras de los camotes de los diferentes grupos experimentales se presentan en el Cuadro 5.

Un análisis de medias Tukey muestra que existió diferencia significativa $(p<0,05)$ entre las variables según el tratamiento. Para la variable L hubo diferencias significativas $(p<0,05)$ entre el empacado en Polietileno de baja densidad y microperforado con relación al resto de los tratamientos $(47,24$ y 45,44 respectivamente). La brillosidad de la cáscara externa disminuye entre más baja sea la humedad relativa a la que está almacenado el camote por la desecación. Así los tubérculos empacados con películas presentan colores más brillantes que los no empacados. Entre los lotes empacados, el microperforado generó camotes con menos brillo que el empacado con polietileno. A
Cuadro 5. Variables de color promedio de la cáscara de los camotes de los diferentes grupos, después de siete días de almacenamiento. San José, Costa Rica. 2007.

\begin{tabular}{|c|c|c|c|c|}
\hline \multirow[t]{2}{*}{ Tratamientos } & \multicolumn{4}{|c|}{ Variables* } \\
\hline & $\mathbf{L}^{1}$ & & $\mathbf{y ~ b}^{3}$ & $\mathbf{a} / \mathbf{b}$ \\
\hline \multirow{2}{*}{$\begin{array}{l}\text { Sin almacenar, sin empaque } \\
\text { (control) }\end{array}$} & \multirow[t]{2}{*}{$44,09 \mathrm{a}$} & $\mathrm{a}$ & 23,87 & \multirow[t]{2}{*}{$3,24 \mathrm{~b}$} \\
\hline & & & 9,13 & \\
\hline \multirow{2}{*}{ Almacenado sin empaque } & \multirow[t]{2}{*}{$43,64 \mathrm{c}$} & $\mathrm{a}$ & 20,23 & \multirow[t]{2}{*}{$2,27 \mathrm{~b}$} \\
\hline & & b & 9,25 & \\
\hline \multirow{2}{*}{ Incubado sin empaque } & \multirow[t]{2}{*}{$42,32 \mathrm{~b}$} & $\bar{a}$ & 16,99 & \multirow[t]{2}{*}{$1,50 \mathrm{a}$} \\
\hline & & U & 11,84 & \\
\hline \multirow{2}{*}{ Incubado + micro-perforado } & \multirow[t]{2}{*}{$45,44 \mathrm{a}$} & $\mathrm{a}$ & 20,28 & \multirow[t]{2}{*}{$2,05 \mathrm{~b}$} \\
\hline & & & 10,41 & \\
\hline \multirow{2}{*}{$\begin{array}{l}\text { Incubado }+ \text { polietileno de } \\
\text { baja densidad }\end{array}$} & \multirow{2}{*}{47,24 a } & $\mathrm{a}$ & 18,83 & \multirow[t]{2}{*}{$1,59 \mathrm{a}$} \\
\hline & & $\mathrm{b}$ & 12,00 & \\
\hline
\end{tabular}

*Los datos seguidos de una misma letra no son estadísticamente diferentes $(\mathrm{p}>0,05)$

${ }^{1}$ luminosidad del color, $0=$ negro y $100=$ blanco.

${ }^{2}$ color entre magenta y verde.

${ }^{3}$ color entre amarillo y azul.

mayor humedad relativa y menor tasa de respiración se dio una menor pérdida de brillo. Como no hubo diferencias significativas los productos sometidos al almacenamiento, estando protegidos por los empaques estudiados, mantuvieron las características de brillo similares al camote fresco.

En el caso del valor $\mathrm{a} / \mathrm{b}$, se detectaron diferencias significativas $(\mathrm{p}<0,05)$ entre el polietileno de baja densidad y el producto sin empacar mantenido en condiciones de ambiente fuera de la cámara. Igualmente hubo diferencia estadística en esta variable $(\mathrm{p}<0,05)$ para ambos lotes de producto sin empacar entre sí, es decir los mantenidos dentro y fuera de la cámara.

El grupo control presentó diferencias significativas $(\mathrm{p}<0,05)$ al compararse los valores $\mathrm{a} / \mathrm{b}$ con el camote en la cámara sin empaque y con el camote empacado en película microperforada. Esta diferencia se manifestó en una disminución de la intensidad de la coloración del camote.

Los tratamientos con camotes sometidos a temperatura ambiente $\left(25^{\circ} \mathrm{C}\right)$, y empacados en una película microperforada, presentaron una coloración más rojiza ( $\mathrm{a} / \mathrm{b}=2,27$ y 2,05 respectivamente), que aquella exhibida por el producto empacado en el polietileno de baja densidad y en la cámara (1,59 y 1,50 respectivamente). 
Es recomendable evaluar a futuro si valores extremos de humedad relativa, como los presentes en ambos tratamientos, ya sea por hidratación o desecamiento de los tejidos, presentan un efecto sobre las antocianinas de la cáscara externa de los camotes, especialmente cuando en un punto intermedio, representado por el microperforado, se dan valores más similares a los camotes frescos.

A partir de los resultados puede afirmarse que sí existió un efecto particular del tipo de empaque y del almacenamiento en el color externo de la cáscara de los camotes, posiblemente asociado con la modificación de las antocianinas y con el proceso de respiración del vegetal.

\section{CONCLUSIONES Y RECOMENDA- CIONES}

La utilización de atmósferas modificadas para emular las condiciones de humedad relativa necesarias durante el almacenamiento en los primeros siete días postcosecha del camote, no implicó un deterioro inaceptable en la calidad general.

Una menor variación de peso y de color se evidenció en los tratamientos almacenados en empaques en comparación con los camotes expuestos al ambiente sin ninguna protección.

Las condiciones de almacenamiento ensayadas, indiferentemente del tratamiento aplicado, no modificaron significativamente la textura original de los camotes frescos.

El color de la pulpa de los camotes distribuidos en los diferentes tratamientos no varió para ninguna de las condiciones de almacenamiento estudiadas; ésto pues no se encontraron diferencias significativas para las variables $\mathrm{L}$ y a/b con respecto a los tratamientos y al camote fresco. Esta variable no solo parece ser muy homogénea entre cultivares como postula la literatura, sino también muy estable entre condiciones de almacenaje.

La brillantez del color de la cáscara disminuyó entre más extrema fue la condición de humedad relativa a la que estuvo almacenado el camote.

Es recomendable estudiar si valores extremos de humedad relativa, ya sea por hidratación o desecamiento de los tejidos, presentan un efecto sobre la pigmentación de la cáscara externa de los camotes.
Los camotes empacados con películas presentaron colores más brillantes que los no empacados, y entre éstos el microperforado generó camotes menos brillantes que el polietileno.

Con base al color final y la textura terminal, el empacado con película microperforada fue el que permitió conservar de manera más apropiada las características prístinas del camote fresco.

Se recomienda la elaboración de futuros trabajos de investigación relacionados con el análisis de la fuerza de penetración en camotes con diferentes métodos de conservación y almacenamiento.

\section{AGRADECIMIENTO}

Se agradece a la M.Sc. Ruth de la Asunción Romero, de la Escuela de Tecnología de Alimentos de la Universidad de Costa Rica, por el apoyo durante la elaboración del presente trabajo.

\section{LITERATURA CITADA}

Afeck, U; Orenstein, J; Nuriel, E. 1998. Increased quality and prolonged storage of sweet potatoes in Israel. Phytoparasitica 26(4):307-312.

Bicudo de Almeida-Muradian, I; De Vouno Camargo Penteado, M; Pupo De Ferreira, V1. 1992. Relationship between carotenoid content and hunter lab colour parameters of Brazilian sweet potato (Ipomea batatas Lam.). Revista Española de Ciencia y Tecnología de Alimentos 32(6): 611-619.

Broadus, EW; Collins, WW; Pharr, DM. 1980. Incidence and severity of hardcore in sweet potatoes as affected by genetic line, curing, and lengths of $1{ }^{\circ} \mathrm{C}$ and $21{ }^{\circ} \mathrm{C}$ storage. Scientia Horticulturae 13(1): 105-113.

Buescher, RW; Sistrunk, WA; Brady, Pl. 1975. Effects of ethylene on metabolic and quality attributes in sweet potato roots. Journal of Food Science 40(5): 10181020.

Chang, LA; Hammett, L; Pharr, DM. 1983. Carbon dioxide effects on ethanol production, pyruvate descarboxylase, and alcohol dehydrogenase activities 
in anaerobic sweet potato roots. Journal of Plant Physiology 71: 59-62.

Collins, JL; Abdul-Aziz, NA. 1982. Sweet potato as an ingredient of yeast raised doughnuts. Journal of Food Science 47(4): 1133-1139.

Collins, JL; Liao, JY; Penfield, MP. 1995. Chemical, physical and sensory attributes of formed and frozen, baked sweet potato. Journal of Food Science 60(3): 465467.

Collins, JL; Washam-Hutsell, L. 1987. Physical, chemical, sensory and microbiological attributes of sweet potato leather. Journal of Food Science 52(3): 646-648, 617 633.

Dizengremel, P; Lance, C. 1976. Control of changes in mitochondrial activities during aging of potato slices. Journal of Plant Physiology 58: 147-151.

Grandberry, DM; Kelley, WT; Boyhan, G. 2005. Sweet potato (en línea). Consultado 03 feb. 2006. Disponible en: http://www.pubs.caes.uga.edu/caespubs/ pubs/PDF/C677.pdf

Heinze, PH; Appleman, CO. 1943. A biochemical study of curing processes in sweet potatoes. Journal of Plant Physiology 18 (4): 548-555.

Hill, B; Roger, TH; Vorhagen, FW. 1997. Comparative analysis of the quantization of color spaces on the basis of the CIELAB color-difference formula. ACM Transactions on Graphics 16(2): 109-154.

Hoover, MW; Walter, WM; Giesbrecht, FG. 1983. Method of preparation and sensory evaluation of sweet potato patties. Journal of Food Science 48(5): 1568-1569.

Huang, YH; Picha, DH; Kilili, AW; Johnson, CE. 1999. Changes in invertase activities and reducing sugars content in sweet potato stored at different temperatures. Journal of Agriculture and Food Chemistry 47(12): 4927-4931.

Instituto Nacional de Investigación y Extensión Agrícola. 2008. Proyecto Camote (en línea). Consultado 19 noviembre de 2008. Disponible. http://www.inia.gob. pe/camote/resumen.htm
James, S. 2005. Sweet potato (en línea). Consultado 03 febrero 2006. Disponible. http://hgic.clemenson.edu/ factsheets/hgic1322.htm

Kay, DE. 1973. Crop and product digest 2: Root Crops. Tropical Products Institute, London. 245 p.

Lerner, R. 2005. The sweet potato (en línea). Consultado 03 febrero 2006. Disponible en: http://www.hort.purdue. edu/ext/HO-136.pdf

LSU Agricultural Center. 2005. Curing and storing sweet potatoes (en línea). Consultado 03 feb. 2006. Disponible en: http://www.lsuagcenter.com/en/crops_ livestock/crops/sweet_potatoes/LSU+AgCenter+Hort iculturist+Discusses+Curing+and+Storing+Sweet+Po tatoes.htm

Mcardle, RN; Bouwkamp, JC. 1986. Use of heat treatments for saccharification of sweet potato mashes. Journal of Food Science 51(2): 364-366.

McConnell, RY; Truoung, VD; Walter, WM; Mcfetters, RF. 2005. Physical, chemical and microbial changes in shredded sweet potatoes. Journal of Food Processing and Preservation 29(1): 246-267.

Motes, JE; Criswell, JT. 2005. Sweet potato production (en línea). Consultado 03 febrero 2006. Disponible en: www.osuextra.com

Mukerjee, PK; Prasad, A. 1972. Storage of sweet potato (Ipomoea batatas Poir). Science \& Culture 38(5): 247-248.

Noda, T; Ohtani, T; Shiina, T; Nawa, Y. 1992. Semi continuous hydrolysis of sweet potato raw starch by Chalara paradoxa glucoamylase. Journal of Food Science 57(6): 1348-1352.

Pangloli, P; Collins, JL; Penfield, MP. 2000. Storage conditions affect quality of noodles with added soy flour and sweet potato. International Journal of Food Science \& Technology 35(2): 235-242.

Picha, DH. 1986. Influence of storage duration and temperature on sweet potato sugar content and chip color. Journal of Food Science 51(1): 239-240. 
Quirien, EA; Ress, D; Aked, J. 2003. Sensory characteristics of five sweet potato cultivars and their changes during storage under tropical conditions. Food Quality and Preference 14(1): 673-680.

Reid, M; Pratt, HK. 1972. Effects of ethylene on potato tuber respiration. Journal of Plant Physiology 49: 252-255.

Saiful-Islam, AFM; Kubota, C; Takagaki, M; Kozai, T. 2002. Sweet potato growth and yield from plug transplants of different volumes, plated intact or without roots. Crop Science 42(1): 822-826.

Schippers, PA. 1977. The rate of respiration of potato tubers during storage. Potato Research 20(2): 173-188.

Singh, RP; Heldman, DR. 1998. Introducción a la ingeniería de alimentos. Acribia. Zaragoza. 544 p.

Sweet Potato First International Symposium (1982 Shanhua, Taiwan) 1982. (Proceedings) Asian Vegetable Research \& Development Center. Shanhua, Taiwan. sp.

Szyperski, RJ; Hamann, DD; Walter, WM. 1986. Controlled alpha amylase process for improved sweet potato puree. Journal of Food Science 51(2): 360-363, 367.
Tanaka, M; Takahata, Y; Nakatani, M. 2005. Analysis of genes developmentally regulated during storage root formation of sweet potato. Journal of Plant Physiology 162: 91-102.

Terahara, N; Konczak-Islam, I; Nakatani, M; Yamakawa, O; Goda, Y; Honda, T. 2000. Anthocyanins in callus induced from purple storage root of Ipomea batatas L. Phytochemestry 54: 919-922

Wang, Y; Horvat, RJ; White, RA; Kays, SJ. 1998. Influence of post harvest curing treatment on the synthesis of the volatile flavor components in sweet potato. Acta Horticulturae 464: 207-212.

Walter, WM; Schadel, W. 1982. Effect of lye peeling conditions on sweet potato tissue. Journal of Food Science 47: 813-817.

Walter, WM; Fleming, HP; Mcfeeters, RF. 1993. Base mediated firmness retention of sweet potato products. Journal of Food Science 58(4): 813-816.

Willis, R; Mcglasso, B; Graham, D; Joyce, D. 1998. Post harvest. 4 ed. Cab International. South Australia. $262 \mathrm{p}$. 
